10-A163 999 THE DISTRIBUTION OF MAXIMUH FLON MITH APPLICATIOW TO HILL CURRICULUA IM OPERATIONS R. G S FISHAMM MOV 85 UNCLASSIFIED UNC/ORSA/TR-85/8 AFOSR-TR-85-125? $F / 812 / 2$
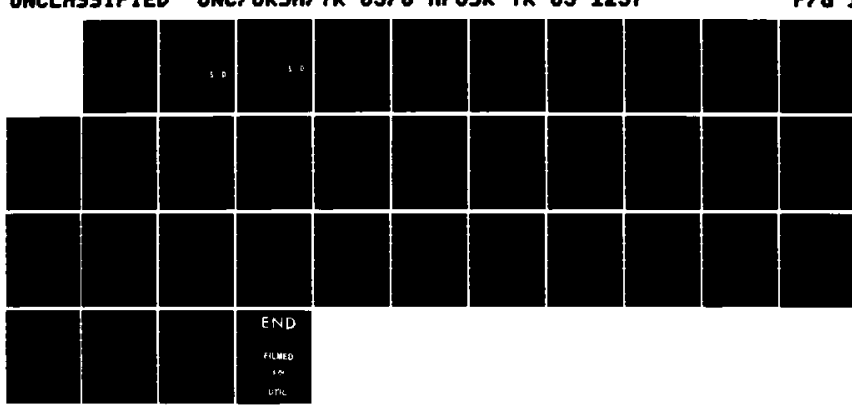

M. 


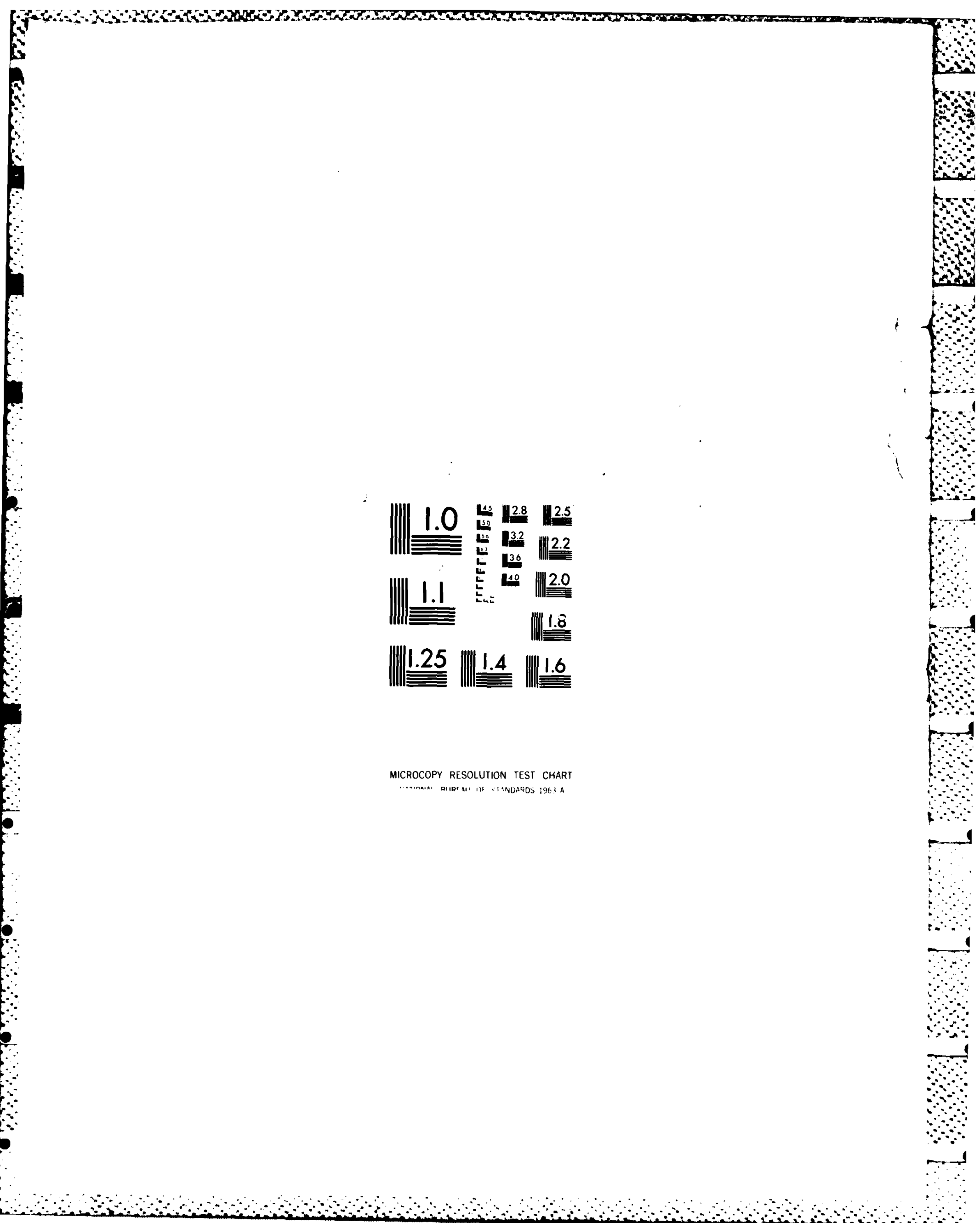


AFOSR - TR $\cdot 2 \div . \overline{257}$

\section{OPERATIONS RESEARCH AND SYSTEMS ANALYSIS}

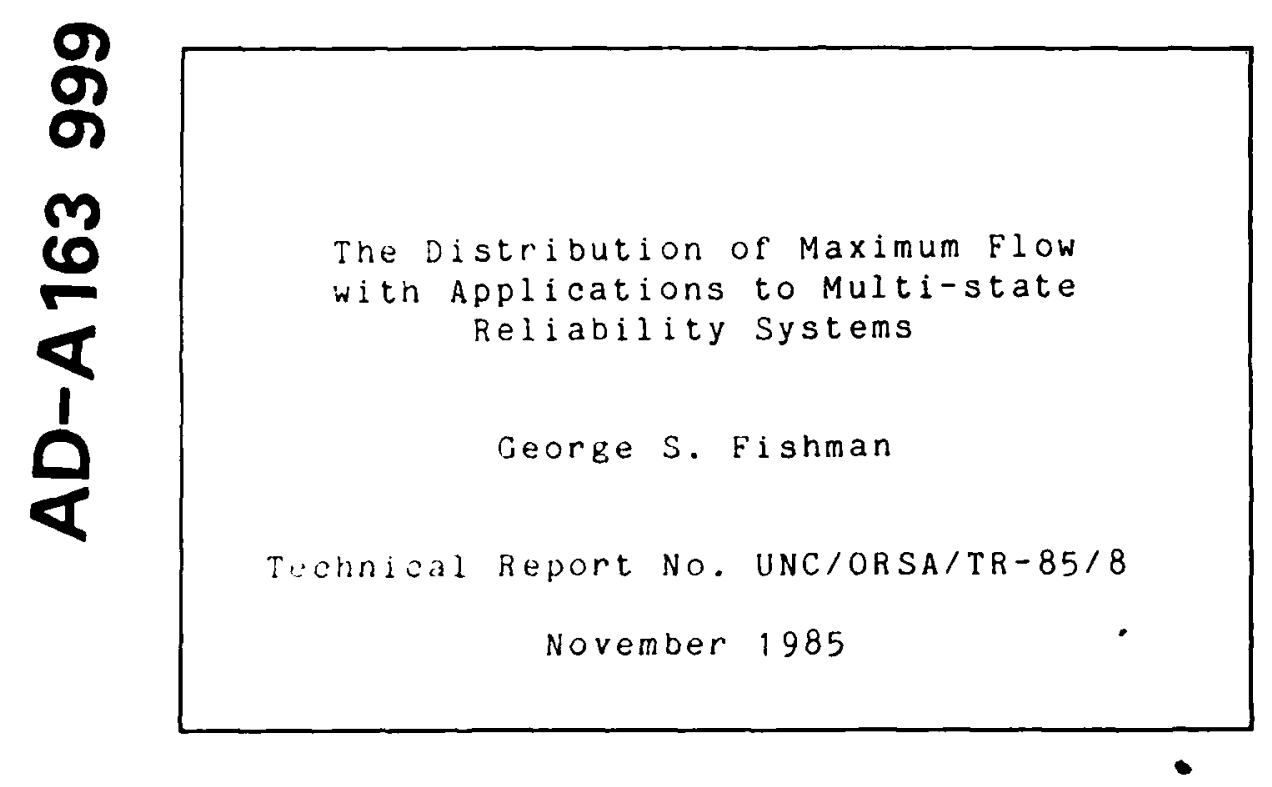

UNIVERSITY OF NORTH CAROLINA AT CHAPEL HILL
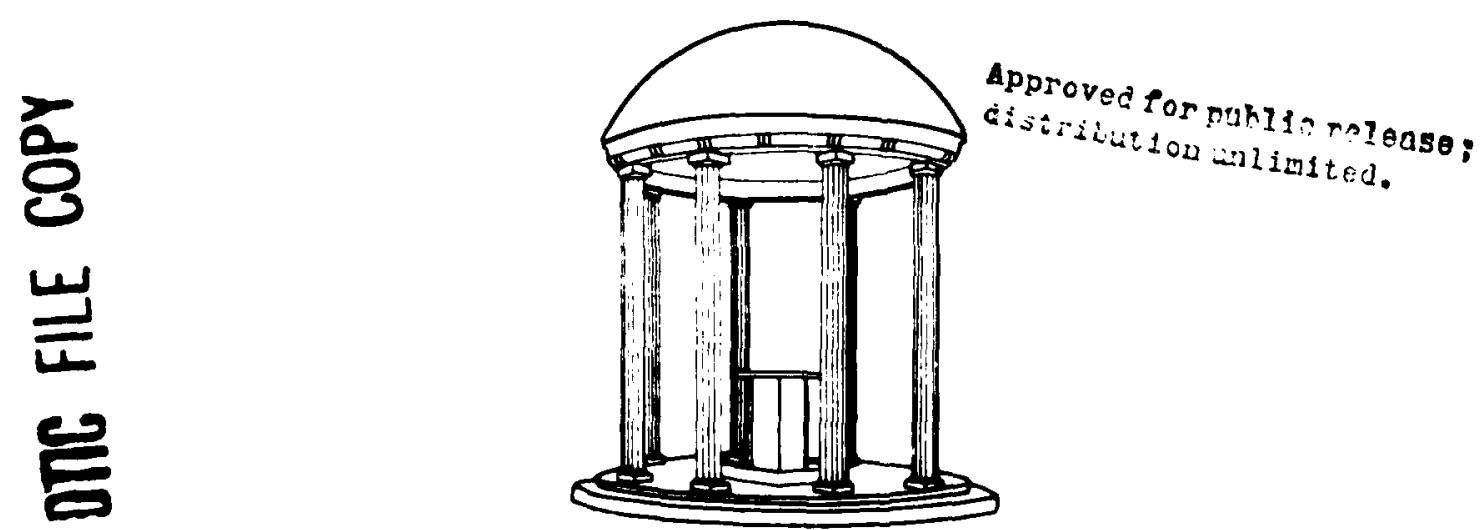


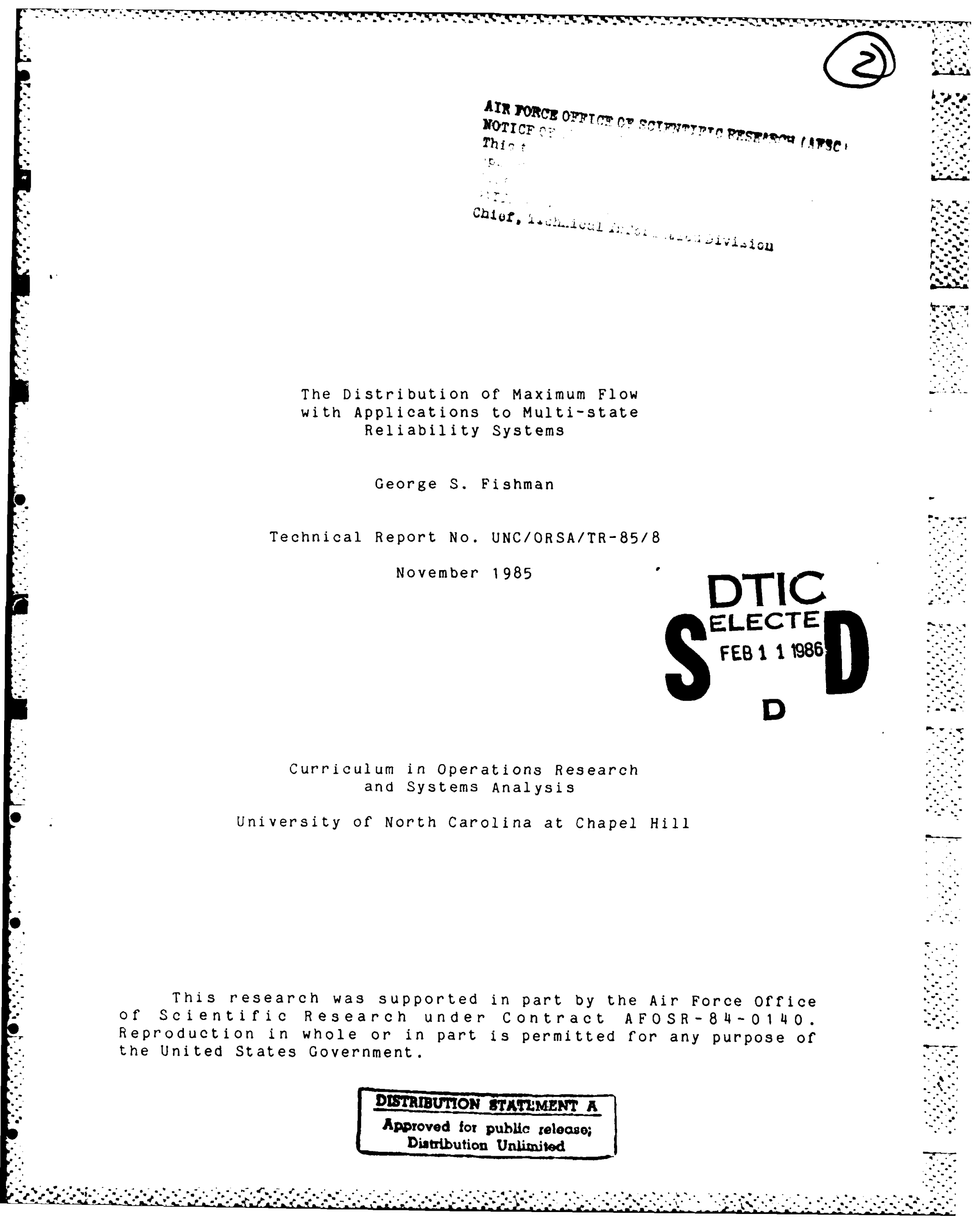




\section{Abstract}

This paper describes an efficient Monte Carlo sampling plan for estimating the distribution of maximum flow in a directed network whose arcs have random capacities. Such a network can be used to represent a multi-state system whose multi-state components are subject to deterioration in capacity by random amounts at random points in time. The proposed sampling plan uses an easily computed a priori upper bound on the complementary distribution function to obtain an unbiased point estimator with smaller variance than crude Monte Carlo sampling allows. The paper also describes procedures for interval estimation and for assessing when the sampling experiment has achieved a specified accuracy. To facilitate sampling, the paper presents a characterization of deterioration based on cumulative processes, leading to the treatment of arc capacities as being multinormaliy distributed. A technique is described for checking the appropriateness of this model with regard to lower and upper bounds on capacity. A procedure is also described for deriving a confidence interval on the measure used to assess variance reduction. An example illustrates the sampling plan and a concise summary gives all steps needed to implement the plan.
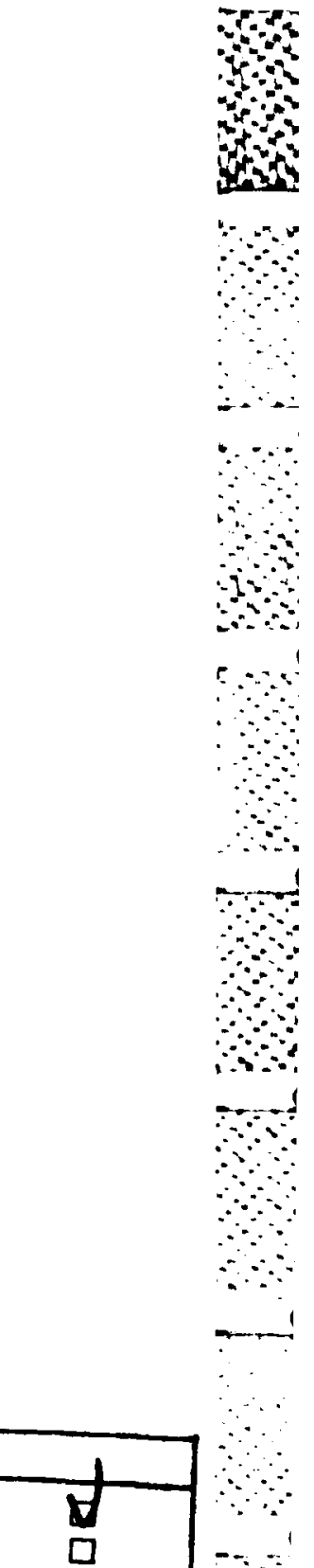

Key words: Maximum flow, Monte Carlo sampling, multi-state reliability

\begin{tabular}{|l|l|}
\hline Availability Codes \\
\hline Dist & $\begin{array}{c}\text { Avail and } \\
\text { Special }\end{array}$ \\
A-/ & \\
\hline
\end{tabular}




\section{Introduction}

This paper describes a method for estimating the complementary distribution function of maximum flow in a multi-state reliability system whose components have capacities that decrease randomly through time and for which maximum flow characterizes system performance. River systems with accumulating silt deposits and distributed manufacturing systems whose individual processing units deterioriate through time provide two examples of such multi-state systems. Although a considerable literature has developed on the reliability of multi-state systems with multi-state components that exhibit random deterioration in performance, this literature mostly concentrates on characterizing properties of performance measures induced by the inherent features of broad classes of multi-state systems. For example, see Barlow and Wu (1978), Baxter and Kim (1984). Block and Savits (1984) and Mak (1985). A considerably more modest literature exists for the equally important topic of computing multi-state performance measures. For example, see Butler (1982), Hudson and Kaper (1985) and Kulkarni and Adlakha $(1985)$.

The present paper is a contribution to this computational literature. Specifically, it characterizes system performance by the concept of maximum flow and describes a Monte Carlo sampling plan for estimating the complementary distribution of maximum flow with considerably better statistical accuracy than crude Monte Carlo sampling would allow for the same cost. For 
convenience of exposition, we use the nomenclature of network analysis to characterize a system. Although the emphasis throughout this paper is on system reliability, the methods described here clearly apply more generally to the considerably broader class of maximum flow problems with capacities subject to random variation.

Let $G=(V, E, s, t)$ denote a directed network with node set $v$, arc set $E=\{1, \ldots, n\}$, source nodes and sink node $t$ $s, t \varepsilon V$. At time $\tau \geq 0$, arcs which represent the components of the system have capacities $B_{1}(\tau), \ldots B_{n}(\tau)$. The capacity of each arc is subject to deterioration so that $B_{j}(\tau) \geq B_{i}(\tau+\Delta) \quad \Delta \geq 0 \quad 1 \leq i \leq n$. The times at which the deteriorations occur are random, as are the magnitudes of the incremental deteriorations themselves. Let $\Gamma$ denote the set of all minimal s-t cutsets of $G$ and define the capacity of cutset $C$ at time $\tau$ as

$$
Z(\tau, C)=\sum_{i \varepsilon C} B_{i}(\tau) \quad C E \Gamma
$$

If capacities were deterministic rather than random, then a measure of system status for each time $\tau$ would be

$$
\Lambda(\tau)=\min _{C \varepsilon \Gamma} Z(\tau, C)
$$

As is well known from the max-flow min-cut theorem of Ford and Fulkerson (1956), $\Lambda(\tau)$ is the maximum possible flow from source node $s$ to sink node $t$ at time $\tau$. Then the function $\{\Lambda(\tau) \tau \geq 0\}$ 
shows the pattern of deterioration in performance in $G$ as time evolves.

When capacities are random, (1) no longer provides an adequate measure of system performance since $\{\Lambda(\tau) \tau \geq 0\}$ is now a stochastic process. Then for fixed $\tau \geq 0$ interest focuses on

$$
L(x, \tau)=\operatorname{pr}[\Lambda(\tau)>x] \quad x \geq 0 .
$$

Also, for fixed $x \geq 0$ interest may focus on the first passage time

$$
\tau_{x}=\min [z: \Lambda(z) \leq x]
$$

for which

$$
\operatorname{pr}\left(\tau_{x}>\tau\right)=L(x, \tau) \text {. }
$$

Then for each $\tau \geq 0\{L(x, \tau), x \geq 0 \mid$ gives the complementary distribution function of maximum flow and for each $x \geq 0\{L(x, \tau)$, $\tau \geq 0$ gives the first passage time complementary distribution function.

other measures of system performance also are of interest. For example,

$$
L(x, C, \tau)=\operatorname{pr}[Z(\tau, C)=\Lambda(\tau) \text { and } \Lambda(\tau)>x]
$$

denotes the probability that $C$ is the critical minimal s-t cutset and that the maximum flow exceeds $x$ at time $\tau$. Then

$$
\begin{aligned}
L(C, \tau \mid x)= & L(x, C, \tau) / L(x, \tau) \\
= & \text { probability that } C \text { is the critical minimal } s-t \\
& \text { cutset, given that the maximum flow exceeds } x \text { at } \\
& \text { time } \tau .
\end{aligned}
$$


Relatively, little work has appeared on the numerical evaluation of the measures (2) through (5). Somers (1982) describes a procedure for computing (2) exactly for the cases of one and two arcs with random capacities and n-1 and $n-2$ arcs with fixed capacities, respectively, and notes that the computation time for $n$ randomly capacitated arcs grows exponentially with $n$. Assuming independent exponentially distributed arc capacities in a planar network, Kulkarni and Adlakha (1985) show how to compute (2) exactly in $O(|V| \cdot|E|)$ time, using the max-flow algorithm of Itai and Shiloach (1979).

Frank and Frisch (1971) provide a comprehensive discussion of the randomly capacitated maximum flow problem with regard to the computation of (2). Their discussion, which is principally in terms of normally distributed arc capacities, gives lower and upper bounds. Because of the difficulty of computation for even moderately sized networks, they suggest a crude Monte Carlo sampling plan to estimate (2).

The present paper describes a Monte Carlo sampling plan for estimating (2) that employs an easily computed upper bound to gain its computational advantage. In particular, this bound enables one to modify sampling in a way that reduces the sampling variation inherent in each trial, thereby reducing the sample size required to achieve a specified accuracy of estimate when compared to crude Monte Carlo sampling. Although the paper concentrates exclusively on (2), one should note that repeated 
application of the proposed sampling plan for a selected set of times $\tau$ would also allow estimation of (3).

To set the stage for the proposed method, Section 1 describes a prototypical crude Monte Carlo sampling plan that remains the basis for comparison throughout the paper. Section 2 presents an improved sampling plan that exploits a priori information on the upper bound to improve statistical efficiency. The plan includes point and interval estimation. Section 3 describes a particular model of component capacity deterioration in a network that enables one to regard capacities on individul arcs as being multinormally distributed. This model greatly facilitates the use of the proposed sampling plan. The section also describes how to assess the appropriateness of the normal model with regard to lower and upper bounds on capacity.

Section 4 presents a procedure to guide the choice of when to stop sampling and section 5 describes inferential methods for assessing the extent of variance reduction. An example in Section 6 illustrates the proposed procedures in detail and Section 7 summarizes the essential steps in sections 2 through 5 to implement the proposed sampling plan.

1. Crude Monte Carlo Sampling

Procedure MFCRUDE describes the steps to be performed in carrying out a crude Monte Carlo sampling plan designed to estinate $\{L(x, \tau) x \in X\}$ from data on $K$ independent trials or 
replications. Here $\overline{\mathrm{L}}_{K}(x, \tau)$ is an unbiased estimate of $L(x, \tau)$ with

$$
\operatorname{var} \bar{L}_{K}(x, \tau)=L(x, \tau)[1-L(x, \tau)] / K
$$

Moreover, $V\left(\bar{L}_{K}(x, \tau)\right)$ in step III is an unbiased estimator of var $\bar{L}_{K}(x, \tau)$. Note that in step I s sapling usually takes $O(n)$ time per replication. However, the dominant time consumer is the determination of maximum flow; for example, taking $0(|V| l o g|V|)$ time per replication for a planar network (Itai and Shiloach $1979)$.

With regard to computing the cell number $j$ in step II, choosing the flow set as $x_{i}=\alpha+\beta i \quad \alpha, \beta>0 i=1, \ldots, r$ offers an advantage. Then for continuous $\Lambda(\tau)$, one has $j=\left\lfloor\min \left\{r,[\Lambda(\tau)-\alpha]^{+/ \beta}\right\}\right\rfloor$ so that the time to determine $j$ is constant and independent of $r$. If the set of flows $x$ does not admit the representation $x_{1}=\alpha+\beta i$, it always will be beneficial to augment the set so that it does.

Procedure MFCRUDE represents a baseline sampling experiment against which one needs to compare any alternative candidate sampling experiment with regard to the presumably smaller resulting variances of interest and the usually larger computing time required per replication to realize these variances. Section 2 describes one such alternative sampling plan based on an easily derivable upper bound on $\{L(x, \tau)\}$. 
Procedure MFCRUDE

Purpose: To estimate (2) for specified time $\tau$ and selected flow levels $x=\left\{x_{0}=0<x_{1}<\ldots<x_{r}\right\}$.

Input: $\quad$ Network $G=(v, E=\{1, \ldots, n\}, s, t)$, time $\tau$, joint distribution of capacities, flow set $x$ and number of independent replications $K$.

output: $\quad\left\{\bar{L}_{K}(x, \tau), V\left(\bar{L}_{K}(x, \tau)\right) ; x \in X\right\}$ as estimators of $\left\{L(x, \tau), \operatorname{var} \bar{L}_{K}(x, \tau) ; x \in X\right\}$, and $\{\Delta S(x) x \in X\}$ as an available input to continued sampling.

Method:

I. Initialization Set $\Delta S\left(x_{i}\right)=0 \quad i=0,1, \ldots, r$.

I . Sampling Experiment

on each of $k$ independent replications: sample the capacities $B_{1}(\tau), \ldots, B_{n}(\tau)$ from the joint distribution; determine the maximum flow $\Lambda(\tau)$; compute $j=\max [i$ : $x_{i}\langle\Lambda(\tau) \quad i=0,1, \ldots, n] ; \Delta S\left(x_{j}\right)=\Delta S\left(x_{j}\right)+1$.

II . Computation of Summary statistics

$$
\begin{aligned}
\bar{L}_{K}\left(x_{i}, \tau\right) & =\frac{1}{K} \sum_{j=i}^{r} \Delta S\left(x_{j}\right) \\
V\left(\bar{L}_{K}\left(x_{i}, \tau\right)\right) & =\bar{L}_{K}\left(x_{i}, \tau\right)\left[1-\bar{L}_{K}\left(x_{i}, \tau\right)\right] /(K-1) .
\end{aligned}
$$

End of Procedure 


\section{$-8-$}

\section{Exploiting Bounds}

The plan to be described here concentrates sampling in a region of the sample space of $B_{1}(\tau), \ldots, B_{n}(\tau)$ where each collected sample outcome contains considerably more information than in the crude Monte Carlo case. Let

$$
H(x, C, \tau)=\{Z(C, \tau)>x\}
$$

so that (2) has the form

$$
\begin{aligned}
L(x, \tau) & =\underset{C \in \Gamma}{\operatorname{pr}\left[\bigcap_{C} H(x, C, \tau)\right]} \\
& =M(x, \tau) N(x, \tau)
\end{aligned}
$$

where for $\Gamma_{1}=\Gamma_{1}(x, \tau) \subseteq \Gamma$

$$
M(x, \tau)=\operatorname{pr}\left[\bigcap_{C \varepsilon \Gamma-\Gamma_{1}} H(x, C, \tau) \mid \bigcap_{C \varepsilon \Gamma \Gamma_{1}} H(x, C, \tau)\right]
$$

and

$$
N(x, \tau)=\operatorname{pr}\left[\bigcap_{C \in \Gamma_{1}} H(x, C, \tau)\right] .
$$

Let

$$
H_{i}(x, \tau)=\bigcap_{C \in \Gamma_{1}} H(x, C, \tau)
$$

and suppose that $N(x, \tau)$ can be computed exactly. Then to estimate $L(X, \tau)$ one proceeds as follows:

on each of $k$ independent replications sample $\underline{B}(\tau)=\left(B_{1}(\tau), \ldots, B_{n}(\tau)\right)$ subject to the restriction $H_{1}(x, \tau)$; determine the maximum flow $\Lambda(\tau)$; set $\phi=I(x, \infty)(\Lambda(\tau))$ where $I_{A}$ denotes the indicator function on the set $A$. 
Then

$$
\bar{M}_{K}(x, \tau)=\frac{1}{K} \sum_{K=1}^{K} \phi(k)
$$

where the superscript $(k)$ denotes trial $k$, is an unbiased estimator of $M(x, \tau)$ and

$$
\bar{L}_{K}(x, \tau)=N(x, \tau) \bar{M}_{K}(x, \tau)
$$

is an unbiased estimator of $L(x, \tau)$ with

$$
\operatorname{var} \tilde{L}_{K}(x, \tau)=L(x, \tau)[N(x, \tau)-L(x, \tau)] / K
$$

Ooserve that

$$
\operatorname{var} \tilde{L}_{K}(x, \tau) \leqq N(x, \tau) / 4 K
$$

which can be a considerably tighter upper bound than that of $1 / 4 \mathrm{k}$ for $\operatorname{var} \bar{L}_{K}(x, \tau)$. More importantly, note that

$$
\frac{\operatorname{var} \bar{L}_{K}(x, \tau)}{\operatorname{var} \tilde{L}_{K}(x, \tau)}=\frac{1-L(x, \tau)}{N(x, \tau)-L(x, \tau)} \geq \frac{1}{N(x, \tau)},
$$

indioating that to achieve

$$
\operatorname{var} \bar{L}_{K_{1}}(x, \tau)=\operatorname{var} \tilde{L}_{K_{2}}(x, \tau)
$$

the ratio $K_{1} / K_{2}$ must be greater than or equal to $1 / N(x, \tau)$. 
The variance ratio in (8) is merely one measure of benefit. Let $\bar{T}$ and $\overline{\mathrm{T}}$ denote the mean times required to perform one replication with crude sampling and with the bound respectively. Then $\bar{T} / \bar{T}$ provides a second measure of performance and is usually less than unity. As a single measure of merit the quantity

$$
R(x, \tau)=\frac{\bar{T}}{\bar{T}} \cdot \frac{\operatorname{var} \bar{L}_{K}(x, \tau)}{\operatorname{var} \bar{L}_{K}(x, \tau)}
$$

is commoniy used in the Monte Carlo literature. This quantity, called the variance reduction ratio, measures the amount of time one would have to run the crude Monte Carlo experiment to achieve the same variance as one would achieve using the proposed method in one unit of time. Clearly $R(x, \tau)>1$ indicates that the proposed method is preferred. Section 5 describes inferential methods of estimating (9) from sample data at the end of an experiment.

Choosing $H_{1}(X, T)$

In selecting $H_{1}(x, \tau)$ one needs to consider several issues. First, $N(x, \tau)$ must be computable. If arc capacities are statistically independent and the cutsets in $\Gamma_{1}(x, \tau)$ are edge-disjoint, then

$$
N(x, \tau)=\pi_{C \in \Gamma_{1}(x, \tau)} \operatorname{pr}[H(x, C, \tau)]
$$


whose computation takes $O\left(\left|\Gamma_{1}(x, \tau)\right|\right)$ time, once the $\operatorname{pr}[H(x, C, \tau)]$ are determined.

To estimate $L(x, \tau)$ for just one flow value $x$, one may want to choose $\Gamma_{1}(x, \tau)$ to be the set of edge-disjoint minimal s-t cutsets that minimizes (10). Unfortunately, the determination of this $r_{1}(x, \tau)$ is NP-complete. Moreover, since one customarily estimates the complementary distribution function for several flow values, it becomes necessary on each replication to insure that $H_{1}(x, \tau)$ holds for each $x$ in $x$. If $I_{1}(x, \tau)$ contalns more than one cutset, this can entail considerable resampling thereby increasing computation time substantially. To reduce this sampling frequency, we describe an alternative method.

Let $\Gamma^{*}$ denote a set of edge-disjoint minimal s-t cutsets, let $x=\left|x_{0}=0<x_{1}<\ldots<x_{r}\right|$ denote the set of flows of interest and define

$$
P_{i}(D, \tau)=\operatorname{pr}\left\{H\left(x_{i}, D, \tau\right) \cap\left[\bigcap_{j=1}^{i-1} H\left(x_{j}, C\left(x_{j}, \tau\right), \tau\right)\right]\right\} \quad D E \Gamma^{*}
$$

where

$$
C\left(x_{1}, \tau\right)=\left\{C: C \in \Gamma^{*} \mid P_{1}(C, \tau) \leq P_{i}(D, \tau) \quad D E \Gamma^{*}\right\}
$$

Here $C\left(x_{1}, \tau\right)$ minimizes $P_{1}(D, \tau)$ at flow $x_{1}$. Now redefine

$$
N\left(x_{i}, \tau\right)=\operatorname{pr}\left[\bigcap_{j=1}^{i} H\left(x_{j}, C\left(x_{j}, \tau\right), \tau\right)\right]
$$

which is again an upper bound on $L\left(x_{1}, \tau\right)$. While it is true that 


$$
N\left(x_{i}, \tau\right) \geq \operatorname{pr}\left[\bigcap_{C \in \Gamma^{*}} H\left(x_{i}, C, \tau\right)\right],
$$

using $\left|C\left(x_{i}, T\right) \quad 1 \leq i \leq r\right|$, as defined in (11), makes resampling necessary for at most one constraint $H\left(x_{i}, C\left(x_{i}, \tau\right), \tau\right)$ at step $i$, in contrast to the potential sampling for the $\left|\Gamma^{*}\right|$ constraints $\bigcap_{C \varepsilon \Gamma^{*}} H\left(x_{i}, C, \tau\right)$ that are active on each step if one chooses to use all cutsets in $\Gamma^{*}$. Note that one can determine a set of edge-disjoint cutsets $\Gamma^{*}=\left|c_{1}, \ldots, c_{J}\right|$ in $O(n)$ time where $J$ is the size of smallest minimal $s-t$ path. Procedure A shows how to deterinine $\left\{C\left(x_{i}, \tau\right) \quad 1 \leq i \leq r\right\}$ given $r^{*}$.

Procedure MFBOUNDS shows how to perform $K$ independent replications to estimate (2) for $\left\{C\left(x_{i}, \tau\right), N\left(x_{1}, \tau\right)\right\}$ as determined in Procedure A. Note that at step i and i constraints $\bigcap_{j=1}^{i} H\left(x_{j}, C\left(x_{j}, \tau\right), \tau\right)$ are in force, although only meeting $H\left(x_{i}, C\left(x_{i}, \tau\right), \tau\right)$ may induce resampling. Since the probability of resampling at step $i \geq 2$ is

$$
1-\frac{\operatorname{pr}\left[Z\left(C\left(x_{i}, \tau\right), \tau\right)>x_{i}\right]}{\operatorname{pr}\left[Z\left(C\left(x_{i}, \tau\right), \tau\right)>x_{j(i)}\right]} \text {. }
$$

where

$$
\begin{aligned}
& j(i)=\max _{1 \leq k<i}\left\{k: C\left(x_{k}, \tau\right)=C\left(x_{i}, \tau\right) \mid \text { if } c\left(x_{i}, \tau\right) \in \mid C\left(x_{k}, \tau\right) \quad i \leq k<i\right\} \\
& =0 \quad \text { otherwise, }
\end{aligned}
$$


the mean number of sampled capacities on each trial in procedure MFBOUND is

$$
\left.n+\sum_{i=2}^{r} c\left(x_{i}, \tau\right)\left\{1-\frac{\operatorname{pr}\left[z\left(c\left(x_{i}, \tau\right), \tau\right)>x_{i}\right]}{\operatorname{pr}\left[z\left(c\left(x_{i}, \tau\right), \tau\right)>x_{j}(i)\right.}\right]\right\}
$$

and an upper bound on the mean number of maximum flow determinations is

$$
r-\sum_{i=2}^{r} \frac{\operatorname{pr}\left[z\left(C\left(x_{i}, \tau\right), \tau\right)>x_{i}\right]}{\operatorname{pr}\left[z\left(C\left(x_{i}, \tau\right), \tau\right)>x_{j(i)}\right]} .
$$

By contrast, Procedure MFCRUDE samples n capacities and determines maximum flow just once per replication. Therefore, Procedure MFBOUNDS clearly takes more time per replication than Procedure MFCRUDE does. 
Procedure A

Purpose: To compute an upper bound for $\{\operatorname{pr}[\Lambda(\tau)>x] x \in X\}$.

Input: $\quad$ Edge-disjoint minimal s-t cutsets $\Gamma^{*}=\left\{c_{1}, \ldots, c_{J}\right\}$,

flow set $x=\left\{x_{0}=0<x_{1}<\ldots<x_{r}\right\}$, time $\tau$ and $\left\{\operatorname{pr}\left[H\left(x_{i}, C_{j}, \tau\right)\right] ; \quad 1 \leq i \leq r, \quad 1 \leq j \leq J\right\}$.

output: $\left\{C\left(x_{i}, \tau\right), N\left(x_{1}, \tau\right) \quad 1 \leq i \leq r\right\}$.

Method: Find the smallest $m$ that minimizes $\operatorname{pr}\left[H\left(x_{1}, C_{m}, \tau\right)\right]$ for all $1 \leq \mathrm{m} \leq \mathrm{J}$; set $\mathrm{C}\left(\mathrm{x}_{1}, \tau\right)=\mathrm{C}_{\mathrm{m}}$; set $\mathrm{N}\left(\mathrm{x}_{1}, \tau\right)=$

$\operatorname{pr}\left[H\left(x_{1}, C\left(x_{1}, \tau\right), \tau\right] ; \quad i=1\right.$.

Until $i=r$ :

A. Find the smallest $m$ that minimizes

$\operatorname{pr}\left\{H\left(x_{i+1}, C_{m}, \tau\right) \cap\left[\bigcap_{j=1}^{i} H\left(x_{j}, C\left(x_{j}, \tau\right), \tau\right)\right]\right\}$ for all $1 \leq m \leq J$;

set $C\left(x_{i+1}, \tau\right)=C_{m}$

set $N\left(x_{i+1}, \tau\right)=N\left(x_{i}, \tau\right) \operatorname{pr}\left[H\left(x_{i+1}, C\left(x_{i+1}, \tau\right), \tau\right)\right]$.

B. If $C_{m} \in\left\{C\left(x_{j}, \tau\right) \quad 1 \leq j \leq i\right\}$ : determine the largest $j$ such that $C\left(x_{j}, \tau\right)=C_{m}$; set

$$
N\left(x_{i}+1, \tau\right)=N\left(x_{i}+1, \tau\right) / \operatorname{pr}\left[H\left(x_{j}, C\left(x_{j}, \tau\right), \tau\right)\right] .
$$

C. $\mathbf{i}=\mathbf{i}+1$.

End of procedure 
Procedure MFBOUNDS

Purpose: To estimate (2) for specified time $\tau$ and selected flow levels $x=\left\{x_{0}=0<x_{1}<\ldots<x_{r}\right\}$.

Input: $\quad$ Network $G=(V, E=|1, \ldots, n|, s, t)$, time $\tau$, joint distribution of capacities, flow set $x$, $\{C(x, \tau), N(x, \tau)$ as determined in Procedure $A ; x \in X\}$ and number of independent replications $K$.

output: $\quad\left\{\tilde{L}_{K}(x, \tau), V\left(\tilde{L}_{K}(X, \tau)\right) ; x \in X\right\}$ as estimators of $\{L(X, \tau)$, $\left.\operatorname{var} \bar{L}_{K}(x, \tau) ; x \in X\right\}$ and $\left\{S(x) \quad x \in X x \neq x_{0}\right\}$, as an available input to continued sampling.

Method:

I. Initialization

Set $C\left(x_{0}, \tau\right)=C\left(x_{1}, \tau\right)$; set $S\left(x_{i}\right)=0 \quad 1 \leq i \leq r$.

I . Sampling Experiment

For each of $K$ independent replications do:

A. Sample capacities $\left\{B_{i}(\tau) \quad 1 \leq i \leq n\right\}$ from the joint distribution subject to the restriction $H\left(x_{1}, C\left(x_{0}, \tau\right), \tau\right)$.

B. Compute maximum flow $\Lambda(\tau)$.

C. Set $z=Z\left(\tau, C\left(x_{0}, \tau\right)\right)$.

D. For $i=1, \ldots, r$ do:

1. $\delta=0$.

2. If $\Lambda(\tau)>x_{i}, \delta=1$. 
3. Otherwise:

$$
\begin{aligned}
& \text { a. If } C\left(x_{i}, \tau\right) \neq C\left(x_{i-1}, \tau\right) \text {, set } Z=Z\left(\tau, C\left(x_{i}, \tau\right)\right) \text {. } \\
& \text { b. If } Z \leq x_{i} \text { : resample }\left\{B_{j}(\tau) j \in C\left(x_{i}, \tau\right)\right\} \text { subject to } \\
& H\left(x_{i}, C\left(x_{i} \tau\right), \tau\right) \text {; set } Z=\sum_{j \in C\left(x_{i}, \tau\right)} B_{j}(\tau) ; \text { compute } \\
& \text { maximum flow } \Lambda(\tau) \text {; if } \Lambda(\tau)>x_{i} \delta=1 .
\end{aligned}
$$

4. $S\left(x_{i}\right)=S\left(x_{i}\right)+\delta$.

II . Compute summary statistics

$$
\begin{gathered}
\tilde{L}_{K}\left(x_{j}, \tau\right)=N\left(x_{j}, \tau\right) S\left(x_{j}\right) / K \\
V\left(\tilde{L}_{K}\left(x_{j}, \tau\right)\right)=N^{2}\left(x_{j}, \tau\right)\left[S\left(x_{j}\right) / K\right]\left[1-S\left(x_{j}\right) / K\right] /(K-1) .
\end{gathered}
$$

End of Procedure

Confidence Intervals

While variances give an indication of statistical accuracy some studies call for the construction of confidence intervals for estimates of interest at a specified level of significance $\alpha \quad 0<\alpha<1$. Let $L=L(x, \tau), N=N(x, \tau)$ and $S=S(x)$ for $x \in X$ where $S(\cdot)$ is given in Procedure MFBOUNDS. Since $S(x)$ has the binomial distribution with parameters $K$ and $L(x, \tau) / N(x, \tau)$, it is in principle possible to derive exact confidence intervals for $L(x, \tau)$. However, as pointed out in detail in Fishman (1984), the 
numerical evaluation of the interval becomes increasingly difficult as $K$ increases. Mere we describe two alternatives.

For binomial samples, the results in okamoto (1958) enable one to show that $\left(N \psi_{1}, N \psi_{2}\right)$ covers $L$ with probability exceeding a where $\psi_{1}<\psi_{2}$ are the solutions of the equation $(K-S) \ln (1-\psi)+S \ln \psi=\ln [(1-\alpha) / 2]+(K-S) \ln (1-S / K)+S \ln (S / K) . \quad(14 a)$ For $\alpha>.9$ these bounds are considerably tighter than those that Chebysher's inequality produces. Also, the interval

$$
\frac{N S+N B^{2} / 2 \pm \beta N\left[\beta^{2} / 4+S(K-S) / K\right]^{1 / 2}}{K+\beta^{2}}
$$

asymptotically $(K \rightarrow \infty)$ covers $L$ with probability $\alpha$ and

$$
B=\left|y:(2 \pi)^{-1 / 2} \int_{-\infty}^{y} e^{-x^{2} / 2} d x=(1+\alpha) / 2\right| .
$$

Employing the tighter normal result requires care. If $L(x, \tau) / N(x, \tau)$ is close to zero or unity then convergence to normality is slow and the normal interval can be misleading. One indicator of the extent of convergence is the skewness measure

$\Omega_{K}=\frac{E\left[\tilde{L}_{K}(x, \tau)-L(x, \tau)\right]^{3}}{\left[\operatorname{var} \tilde{L}_{K}(x, \tau)\right]^{3 / 2}}=\frac{N(x, \tau)-2 L(x, \tau)}{\{K L(x, \tau)[N(x, \tau)-L(x, \tau)]]^{1 / 2}}$

A skewness close to zero supports the contention of normality. Section 6 uses a sample value for $\Omega_{K}$ to make this assessment. 
occasionally it may be of interest to compute simultaneous confidence intervals for, say, $\left\{L(X, T) x \in X^{*}\right\}$ where $X^{*} \subseteq X$ is a finite set of flows. Then the $\left|x^{*}\right|$ okamoto confidence intervals that result from (14a) hold simultaneously with probability of at least $\alpha$ when $1-(1-\alpha) /\left|x^{*}\right|$ replaces $\alpha$ in (14a). Also, confidence intervals based on normality (14b) hold asymptotically when this same substitution for $\alpha$ is made in (15). These simultaneous interval results follow from a Bonferroni inequality. See Miller $(1981$, p. 8$)$.

\section{Characterizing Deterioration Through Time}

At least two distinct characterizations exist for multi-state systems with random capacities for which maximum flow is a performance measure. One representation views system components as experiencing successive moderate-to-small capacity reductions of random magnitude at a sequence of random points in time. The second views the components as either operating at full capacity or as failed at random points in time. The present paper focuses on the first characterization and adopts a formulation from the theory of cumulative processes, as described in Smith (1955), to facilitate the estimation of $\{L(x, \tau)\}$.

Let ij denote the time at which the ith capacity reduction occurs to component $j$ so that $\tau_{i j}<\tau_{k j} k=i+1, i+2, \ldots$ Assume that $\left.\mid \tau_{i j}-\tau_{i-1, j} \quad \tau_{0 j}=0 ; \quad i=1,2, \ldots\right\} \quad j=1, \ldots, q$ form $q$ independent stationary renewal processes with 


$$
E\left(\tau_{i j}-\tau_{i-1, j}\right)<\alpha_{j}<\infty
$$

and

$$
i=1,2, \ldots ; j=1, \ldots, q \text {. }
$$

$$
\operatorname{var}\left(\tau_{i j}-\tau_{i-1, j}\right)=B_{j}<\infty
$$

We conceive of $\tau_{i j}$ as the time of the ith degradation for arci in arc set $E_{j}$ where

$$
\begin{aligned}
E_{j} \cap E_{k} & =\emptyset \quad j \neq k \\
E & =E_{1}+\ldots+E_{q} .
\end{aligned}
$$

Define

$$
\begin{aligned}
A_{i j}= & \text { reduction in capacity on arc } j \text { at } \\
& \text { time } \tau_{1 j}
\end{aligned}
$$

and for each $j$ take $A_{1 j}, A_{2 j} \ldots$ to be independent and identically distributed (i.i.d.) random variables with

$$
\begin{aligned}
E A_{i j} & =Y_{j}<\infty \\
\operatorname{var} A_{i j} & =\lambda_{j}<\infty \\
\operatorname{corr}\left(A_{i j}, A_{i k}\right) & =\rho_{j k} \quad \text { if } \quad j \in E_{k} \\
& =0 \quad \text { otherwise }
\end{aligned}
$$

and

$$
\begin{array}{rlrl}
\operatorname{corr}\left(A_{i j}, \tau_{i k}-\tau_{i-1, k}\right) & =\omega_{j} \quad \text { if } j \in E_{k} \\
& =0 & \text { otherwise. }
\end{array}
$$

Let

$$
J\left(\tau, E_{k}\right)=\text { number of degradation times in }[0, \tau] \text { in set } E_{k}
$$
so that at time $t$ the accumulated degradation on arc $j$ in set $E_{k}$ is 


$$
\begin{aligned}
& -20- \\
& \begin{array}{rlrl}
Y_{j}(\tau) & =\sum_{i=1}^{J\left(\tau, E_{k}\right)} A_{i j} & \text { if } J\left(\tau, E_{k}\right)>0 \\
& =0 & & \text { otherwise }
\end{array}
\end{aligned}
$$

and the capacity of arc $j$ is

$$
\begin{aligned}
B_{j}(\tau) & =B_{j}(0)-Y_{j}(\tau) . \\
\text { As } \tau \rightarrow \infty, \text { one has } & (\operatorname{Smith} 1955) \text { for arcs } j, m \in E_{k} \\
Y_{j}(\tau) & =\mu_{j} \tau+O(\tau),
\end{aligned}
$$

where $O(\tau)$ denotes a function $h(\tau)$ such that $l$ im $h(\tau) / \tau=0$,

$$
\begin{aligned}
\mu_{j} & =\gamma_{j} / \alpha_{k}, \\
\operatorname{var} Y_{j}(\tau) & =\sigma_{j j} \tau+o(\tau), \\
\sigma_{j j} & =\left[\lambda_{j}-2 \omega_{j}\left(\gamma_{j} / \alpha_{k}\right)\left(\beta_{k} \lambda_{j}\right)^{1 / 2}+\beta_{k}\left(\gamma_{j} / \alpha_{k}\right)^{2}\right] / \alpha_{k},
\end{aligned}
$$

and

$$
\begin{array}{rlrl}
\operatorname{cov}\left[Y_{j}(\tau), Y_{m}(\tau)\right] & =\sigma_{j m} \tau+o(\tau) & \text { if } j \varepsilon E_{m} \\
& =0 & & \text { otherwise }
\end{array}
$$

where

$\sigma_{i j}=\left[\rho_{j m}\left(\lambda_{j} \lambda_{m}\right)^{1 / 2}-\omega_{j} \gamma_{j}\left(\beta_{k} \lambda_{j}\right)^{1 / 2 / \alpha_{k}}-\omega_{m} \gamma_{m}\left(\beta_{k} \lambda_{m}\right)^{2 / 2} / \alpha_{k}+\beta_{k} \gamma_{j} \gamma_{k} / \alpha_{k}^{2}\right] / \alpha_{k}$.

Moreover, as $t \rightarrow \infty$ the joint distribution of 
$\left\{\left[B_{j}(\tau)-B_{j}(0)+\mu_{j} \tau\right] / \tau^{1 / 2} j \varepsilon E\right\}$ converges to the multinormal distribution with mean vector $\stackrel{0}{a}$ and covariance matrix $\Sigma=\left\|\sigma_{i j}\right\|$. Then, for large $\tau\{z(\tau, C) C \varepsilon \Gamma\}$ are approximately normal with

$$
E Z(\tau, C)=\sum_{j \in C}\left[B_{j}(0)-\mu_{j} \tau\right]
$$

and C, DE Г .

$$
\operatorname{cov}[Z(\tau, C), Z(\tau, D)]=\tau \sum_{i, j \in C \cap D} \sigma_{i j}
$$

When this formulation in terms of cumulative processes applies, it offers many conveniences for Monte Carlo sampling. observe that, because of the asymptotic normality, the sampling distribution of $B_{1}(\tau), \ldots, B_{n}(\tau)$ requires knowledge only of the initial capacities $\left|B_{i}(0) \quad 1 \leq i \leq n\right|$, the means $\left\{\mu_{i} 1 \leq i \leq n\right\}$ and the covariances $\left\{\sigma_{i j} 1 \leq i, j \leq n\right\}$. In particular, note that the covariances $\left|\sigma_{i j} i \neq j\right|$ completely characterize statistical dependence in capacity changes on different arcs. It is entirely plausible that one can derive suitable empirical estimates of these quantities from time series or cross-sectional data for multi-state systems encountered in practice. Moreover, sampling $\left\{B_{j}(\tau) j \varepsilon C \mid\right.$ subject to $H(x, C, \tau)$ is easily realized using the conditional properties of the normal distribution and Algorithm RSNB in Cheng (1981,pp.314-315), which takes $O(|C|)$ time.

Two restrictions need to be kept in mind when assessing the sultability of this probabilistic model. They are 


$$
\max _{j \varepsilon E}\left[B_{j}(\tau)-B_{j}(0)\right] \leq 0
$$

and

$$
\min _{j \in E} B_{j}(\tau) \geqq 0
$$

which reality demands with probability one but to which normal theory assigns some positive probability less than unity. In the case of statistically independent capacities this probability is $1-\theta(\tau)$ where

$$
\theta(\tau)=1-\prod_{j=1}^{n}\left[\Phi\left(\frac{B_{j}(0)-\mu_{j} \tau}{\left(\sigma_{j j} \tau\right)^{1 / 2}}\right)-\Phi\left(-\mu_{j}\left(\tau / \sigma_{j j}\right)^{1 / 2}\right)\right]
$$

and $\Phi(\cdot)$ denotes the standard normal distribution function. Clearly 1 - $\theta(\tau)$ should be small before one accepts the suitability of the normal approximation. As an additional precaution when using the normal model one can use $\max \left\{0, \min \left[B_{j}(0), B_{j}(\tau)\right]\right\}$ in place of $B_{j}(\tau)$ for the capacity of arc $j$ at time $\tau$ in Procedure MFBOUNDS.

\section{Credibility Analysis}

There remains the question of how large $k$ should be in order to ensure the level of statistical accuracy required for a particular study. As examples, one may want to estimate $L(x, \tau)$ subject to the absolute error criterion

$$
I_{1}(x, \varepsilon)=\left\{\left|\tilde{L}_{K}(x, \tau)-L(x, \tau)\right| \leqq \varepsilon\right\} \quad \varepsilon>0
$$

or the relative error criterion 


$$
I_{2}(x, \omega)=\left\{\left|\tilde{L}_{K}(x, \tau)-L(x, \tau)\right| \leq \omega L(x, \tau)\right\} \quad 0<\omega<1 .
$$

Some studies may demand that the least criterion

$$
I_{3}(x, \varepsilon, \omega)=I_{1}(x, \varepsilon) \cup I_{2}(x, \omega)
$$

be satisfied and others may demand that both criteria

$$
I_{4}(x, \varepsilon, w)=I_{1}(x, \varepsilon) \cap I_{2}(x, w)
$$

be met. Here $\mathrm{I}_{3}(x, \varepsilon, \omega)$ is the least stringent and $\mathrm{I}_{4}(x, \varepsilon, \omega)$ is the most stringent.

Since $S(x)$ in Procedure MFBounds has the binomial distribution with parameters $K$ and

$$
\mu(x)=L(x, \tau) / N(x, \tau) \text {. }
$$

satisfying $I_{1}(x, \varepsilon, \omega)=I_{1}(x, \varepsilon)$ is equivalent to requiring that $\mu(x) \in\left[\mu_{1 *}(x), \mu_{1}^{*}(x)\right]$, where

$$
\mu_{1 *}(x)=[S(x) / K-\varepsilon / N(x, \tau)]^{+}
$$

and

$$
\mu_{1}^{*}(x)=1-[1-S(x) / K-\varepsilon / N(x, \tau)]^{+},
$$

and satisfying $I_{2}(x, \varepsilon, \omega)=I_{2}(x, \omega)$ is equivalent to requiring that $\mu(x) \in\left[\mu_{2 *}(x), \mu_{2}^{*}(x)\right]$ where

$$
\mu_{2 *}(x)=S(x) / K(1+w)
$$

and 


$$
\mu_{2}^{*}(x)=1-[1-S(x) / K(1-\omega)]^{+}
$$

Moreover, satigfying $I_{3}(x, \varepsilon, \omega)$ implies $\mu(x) \varepsilon\left[\mu_{3}(x), \mu_{3}^{*}(x)\right]$, where

$$
\mu_{3 *}(x)=\min \left[\mu_{1 *}(x), \mu_{2 *}(x)\right]
$$

and

$$
\mu_{3}^{*}(x)=\max \left[\mu_{1}^{*}(x), \mu_{2}^{*}(x)\right] \text {, }
$$

and satisfying $I_{4}(x, \varepsilon, \omega)$ implies $\mu(x) \varepsilon\left[\mu_{4 *}(x), \mu_{4}^{*}(x)\right]$ where

$$
\mu_{4 *}(x)=\max \left[\mu_{1 *}(x), \mu_{2 *}(x)\right]
$$

and

$$
\mu_{4}^{*}(x)=\min \left[\mu_{1}^{*}(x), \mu_{2}^{*}(x)\right]
$$

That is, for $S=S(x)$

$$
\begin{aligned}
1-\operatorname{pr}\left[I_{j}(x, E, \omega)\right] & =\operatorname{pr}\left\{\mu(x) \neq\left[\mu_{j *}(x), \mu_{j}^{*}(x)\right]\right\} \\
& =1-F_{S}\left(K, \mu_{j *}(x)\right)+F_{S}\left(K, \mu_{j}^{*}(x)\right) \quad 1 \leq j \leq 4 .
\end{aligned}
$$

F being the binomial distribution function.

For sets of flows $x=\left|x_{1}<\ldots<x_{r}\right|$, absolute errors $\left|\varepsilon_{1}, \ldots, \varepsilon_{r}\right|$ and relative errors $\left\{\omega_{1}, \ldots, \omega_{r}\right\}$.

$$
1-\operatorname{pr}\left[\bigcap_{i=1}^{r} I_{j}\left(x_{i}, \varepsilon_{i}, w_{i}\right)\right] \leq A_{j}
$$

where 


$$
A_{j}=\sum_{i=1}^{r}\left[1-F_{S}\left(K, \mu_{j *}\left(x_{i}\right)\right)+F_{S}\left(K, \mu_{j}^{*}\left(x_{i}\right)\right)\right] 1 \leq k \leq 4,
$$

the upper bound following again from a Bonferroni inequality (Tong 1980, p.143).

To guide the choice of sample size, one can increase $k$ in steps, compute the bound corresponding to the chosen criterion at the end of each step and use the sequence of such values as a measure of the credibility of having satisfied the criterion. To ensure some progress in the value of the bound, increments in $k$ should be relative. Doubling $k$ at each step is a reasonable choice. IMSL (1982) and SAS (1982) provide routines for evaluating the binomial distribution with high accuracy. Note that in contrast to the discussion on confidence intervals in Section 3, the development here relies on the exact binomial distribution.

\section{Estimating Variance Reduction}

As section 3 notes, (9) indicates the benefits of variance reduction. One can estimate this quantity by

$$
\bar{R}(x, \tau)=\frac{\overline{\mathrm{T}}}{\overline{\mathrm{T}}} \times \bar{Q}(x, \tau)
$$

where

$$
\tilde{Q}(x, \tau)=\left[1-\tilde{L}_{K}(x, \tau)\right] /\left[N(x, \tau)-\tilde{L}_{K}(x, \tau)\right] \text {. }
$$


$\overline{\mathrm{T}}$ and $\overline{\mathrm{T}}$ being the sample mean times per replication for Procedures MFCRUDE and MFBOUNDS respectively. Although $\bar{T} / \bar{T}$ generally is a relatively stable quantity, $\bar{Q}(x, \tau)$ may not be, especially when $L(x, \tau)$ is close to $N(x, \tau)$. Therefore, relying solely on (26) as an indicator of variance reduction can be misleading. To resolve this problem, one can compute a confidence interval for $R(x, \tau)$ in $(9)$.

$$
\text { Let } \begin{aligned}
N=N(x, \tau), \quad & =\tilde{L}_{K}(x, \tau) \text { and } R=R(x, \tau), \text { so that } \\
Y & =1-\tilde{L}-R(N-\bar{L})
\end{aligned}
$$

has mean zero and

$$
\operatorname{var} Y=(N R-1)(1-N) / K
$$

Then Chebyshev's inequality gives

$$
\operatorname{pr}\left[\frac{|1-\tilde{L}-R(N-\tilde{L})|}{[(N R-1)(1-N) / K]^{1 / 2}}<\frac{1}{(1-\alpha)^{1 / 2}}\right]>\alpha
$$

yielding a $100 \times \alpha$ confidence interval

$\frac{(1-\tilde{L})(N-\bar{L})+\beta^{2} N(1-N) / 2 K \pm \beta(1-N)\left[(N-\tilde{L}) \tilde{L} / K+\beta^{2} N^{2} / 4 K^{2}\right]^{1 / 2}}{(N-\tilde{L})^{2}}$

with $\beta=1 /(1-\alpha)^{1 / 2}$. If warranted, a tighter normal interval with $B$ computed as in (15) can be used.

6. Example

Figure 1 shows a directed network for which we wish to estimate $\{L(X, \tau)\}$ for $T=500$ and $X=|800+1001 \quad 1=1, \ldots, 12|$. 
Table 1 gives the corresponding arc parameters. Since Insert Fig. 1 and Tables 1,2 and 3 about here.

$\theta(500)=.0172$ in $(20)^{\prime}$ the normal model is reasonable. The selected set of edge-disjoint cutsets is $c_{1}=\{1,2,3\}$, $c_{2}=\{14,24,25\}$, and $c_{3}=\{5,6,9,10,12\}$. Table 2 shows $\left\{C\left(x_{i}, \tau\right)\right.$, $\left.N\left(x_{i}, \tau\right) \quad 1 \leq i \leq r\right\}$. Table 3 shows the results of using Procedure MFBOUNDS for $K=65536$ independent trials. In addition to the favorable variance reduction for all $x$, note the wide confidence intervals for $R(x, \tau)$ for $900 \leq x \leq 1400$, reflecting the closeness of the upper bound $N(x, \tau)$ to $L(x, \tau)$. Also, note the substantial variance reduction for $x \geq 1800$ and the fact that variance reduction is not monotone in $x$.

Figure 2 provides an understanding of this nonmonotone behavior. These cutsets act as the principal restrictions on

Insert Fig. 2 about here.

capacity when looking at flows in the interval [0, 2000]. For small $x, C_{1}$ is the dominant restriction. As $x$ increases $C_{1}$ and $\mathrm{C}_{2}$ become equally dominant and, as $x$ continues to increase, $\mathrm{C}_{2}$ becomes the restrictive cutset. The point of lowest variance reduction apparently occurs where $C_{1}$ and $C_{2}$ are approximately equally dominant. 
Table 4 gives individual okamoto and normal. 95 confidence intervals for $\left\{L\left(x_{j}, \tau\right) 1 \leq i \leq r\right\}$ along with the sample skewness based on (16). Although both sets of intervals give at least two digit accuracy, the sample skewness encourages one to rely more on the okamoto bounds for $900 \leq x \leq 1300$.

Insert Tables 4 and 5 about here.

To demonstrate the credibility analysis of section 4 , Table 5 lists the bounds for the four criteria described there for $\varepsilon_{i}=\varepsilon=.01$ and $\omega_{i}=\omega=.11 \leq i \leq r$ for Procedure MFBOUNDS and for crude Monte Carlo sampling in Procedure MFCRUDE. Most notable is the relative error criterion which shows that a bound less than .1 is achieved with high certainty at $k=4096$ in contrast to Procedure MFCRUDE which does not achieve this bound with moderate assurance even for $K=1048576$. That is, MFCRUDE requires a sample size at least 1024 times larger than that for MFCRUDE to achieve the same relative error criterion.

7. Essential Steps for Implementation

Here we list in appropriate order the essential steps needed to implement the proposals in this paper:

1. Select the time $\tau$ of interest.

2. Select the flow set $x=\left\{x_{0}=0<x_{1}<\ldots<x_{r}\right\}$. 
3. Compute $\theta(\tau)$ in (20) to check the appropriatenesss of the normal model. If inappropriate, stop.

4. Select an error criterion and specify the corresponding $\left\{\varepsilon_{1} \ldots, \varepsilon_{r}\right\}$ and $\left\{\omega_{1}, \ldots \omega_{r}\right\}$ (section 4$)$.

5. Determine a set of edge-disjoint cutsets $\Gamma^{*}=\left|c_{1}, \ldots, c_{J}\right|$.

6. For each $x \in X$, determine $C(x, \tau)$ and $N(x, \tau)$ using Procedure A.

7. Use Procedure MFBOUNDS for the sampling experiment.

8. If the experiment is to be performed in blocks of $K_{1}, K_{1}+K_{2}, K_{1}+K_{2}+K_{3} \ldots$ replications, then after each block compute the credibility probability bound for the selected error criterion. Suggested increments are $K_{i}=K_{1} 2^{i-1}$ for $i=1,2, \ldots$

9. After completion of the experiment compute the sample skewness in (16) and a confidence interval for each $L(x, \tau) \quad x \in X \quad x \neq x_{0} \cdot$

Acknowledgements

I would like to express my gratitude to J. Scott provan for our many helpful discussions and to Tien yi shaw for his able programming assistance. 
References

1. Barlow, R. E. and A. S. Wu (1978). Coherent systems with multi-state components, Mathematics of operations Research, $3,275-281$.

2. Baxter, L. A. and C. Kim (1984). Modules of continuum structures, presented at Conference on Reliability Theory and Quality Control, University of Missouri-Columbia, June 1984 .

3. Block, H. and T. H. Savits (1984). Continuum multi-state structure functions oper. Res., 32, 703-714.

4. Butler, D. A. (1982). Bounding the reliability of multi-state systems, Operations Research, 30, 530-544.

5. Cheng, R. C. H. (1981). The use of antithetic variates in computer simulators, Proceedings of the Winter simulation Conference, T. I. Oven, C. M. Delfosse and C. M. Shub eds.

6. Fishman, G. S. (1984). A Monte Carlo sampling plan for estimating network reliability, Technical Report No. UNC/ORSA/TR-84/8, Curriculum in operations Research and Systems Analysis, University of North Carolina at Chapel Hill.

7. Ford, L.R., Jr. and D. R. Fulkerson (1962). Flows in Networks, Princeton University Press.

8. Frank, H. and I. T. Frisch (1971). Communication Transmission and Transport Networks, Addison-Wesley. Reading, Massachusetts.

9. Hudson, J.C. and K.C. Kapur (1985). Reliability bounds for multi-state systems with multi-state components, oper. Res., $33,153-160$.

10. IMSL, Inc. (1982). IMSL Library Reference Manual, Edition 8 , Houston, Texas.

11. Itai, A. and Y. Shiloach (1979). Maximum flow in planar networks, SIAM J. Comput.. 8, 135-150.

12. Kulkarni, V.G. and V. G. Adlakha (1984). Maximum flow in planar networks with exponentially distributed arc capacities, Technical Report UNC/ORSA/TR-84/15, Curriculum in operations Research and Systems Analysis, University of North Carolina at Chapel Hill. 
13. Mak, King-Tim (1985). Coherent continuous system and generalized associativity function equation, University of Illinois at Chicago, P. 0. Box 4348, Chicago, Il. 60680.

14. Miller, R. (1981). Simultaneous Statistical Inference, Springer Verlog, second edition.

15. Okamoto, M. (1958). Some inequalities relating to the partial sum of binomial probabilities, Annuals of the Institute of Statistical Mathematics, 10, 29-35.

16. Papadimitriou, C. H. and K. Steiglitz (1982). Combinatorial optimization: Algorithms and Complexity, Prentice Hall, Englewood Cliffs, New Jersey.

17. SAS Institute, Inc. SAS User's Guide: Basics, Cary, North Carolina.

18. Smith, W.L. (1955). Regenerative stochastic processes,

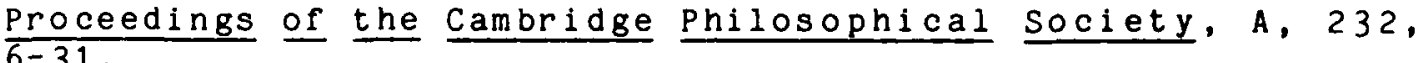

19. Somers, J.E. (1982). Maximum flow in networks with a small number of random arc capacities, Networks, 12, 241-253.

20. Tong, Y. L. (1980). Probability Inequalities in Multivariate Distributions, Academic Press.

21. Tsukiyama, S., I. Shirakawa, H. Ozaki and H. Ariyoshi (1980). An algorithm to enumerate all cutsets of a graph in linear time per cutset, J. ACM, 27, 619-632. 
Table 1

Capacity Parameters for

Network in Figure 1

arc j

$B_{j}(0)$

$\mu_{j}$

${ }^{\sigma} j \mathrm{j}$

868.33

2532.62

1736.66

3473.31

3618.03

723.61

3256.23

2170.82

1736.66

723.61

868.33

3256.23

1302.49

1809.02

863.33

2604.98

3618.03

2894.43

1085.41

1447.21

3256.23

2532.62

1809.02

1736.66

2170.82
1.20

3.50

2.40

4.80

5.00

1.00

4.50

3.00

2.40

1.00

1.20

4.50

1.80

2. 50

1.20

3.60

5.00

4.00

1.50

2.00

4.50

3.50

2. 50

2.40

3.00
14.40

122.50

57.60

230.00

250.00

10.00

202.50

90.00

57.60

10.00

14.40

202.50

32.40

6.25

14.40

129.60

250.00

160.00

22.50

4.00

202.50

122.50

62.50

57.60

9.00 
Table 2

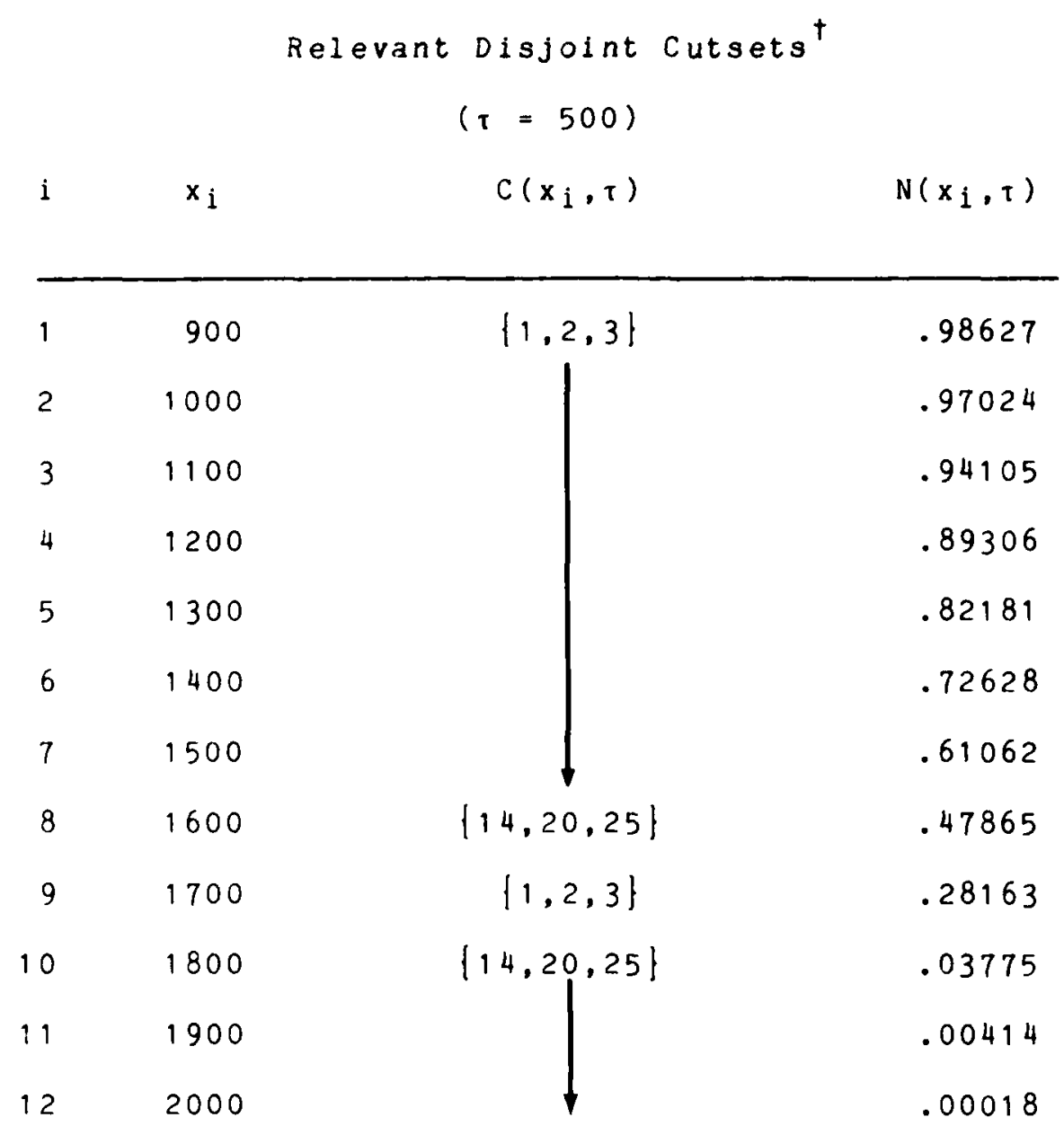

$+\left\{C\left(x_{i}, \tau\right)\right\}$ is computed as in (11). $\left\{N\left(x_{i}, \tau\right)\right\}$ is computed as in (12). 
Table 3

Experimental Results ${ }^{\dagger}$

$(\tau=500, K=65536, \overline{\mathrm{T}} / \overline{\mathrm{T}}=.335)$

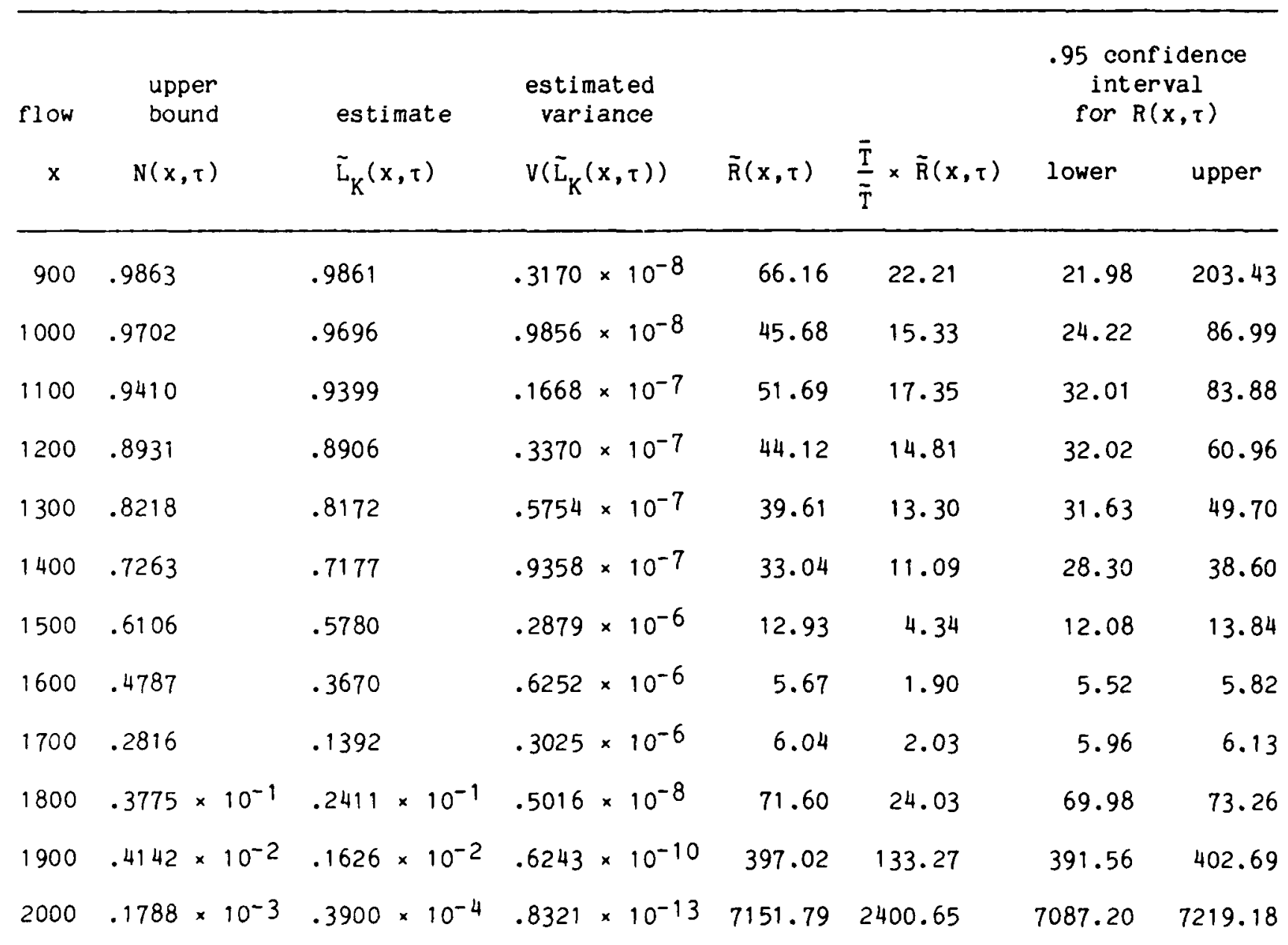

$\dagger_{\overline{\mathrm{T}}}$ and $\overline{\mathrm{I}}$ were obtained from runs with MFCRUDE and MFBOUNDS respectively. 


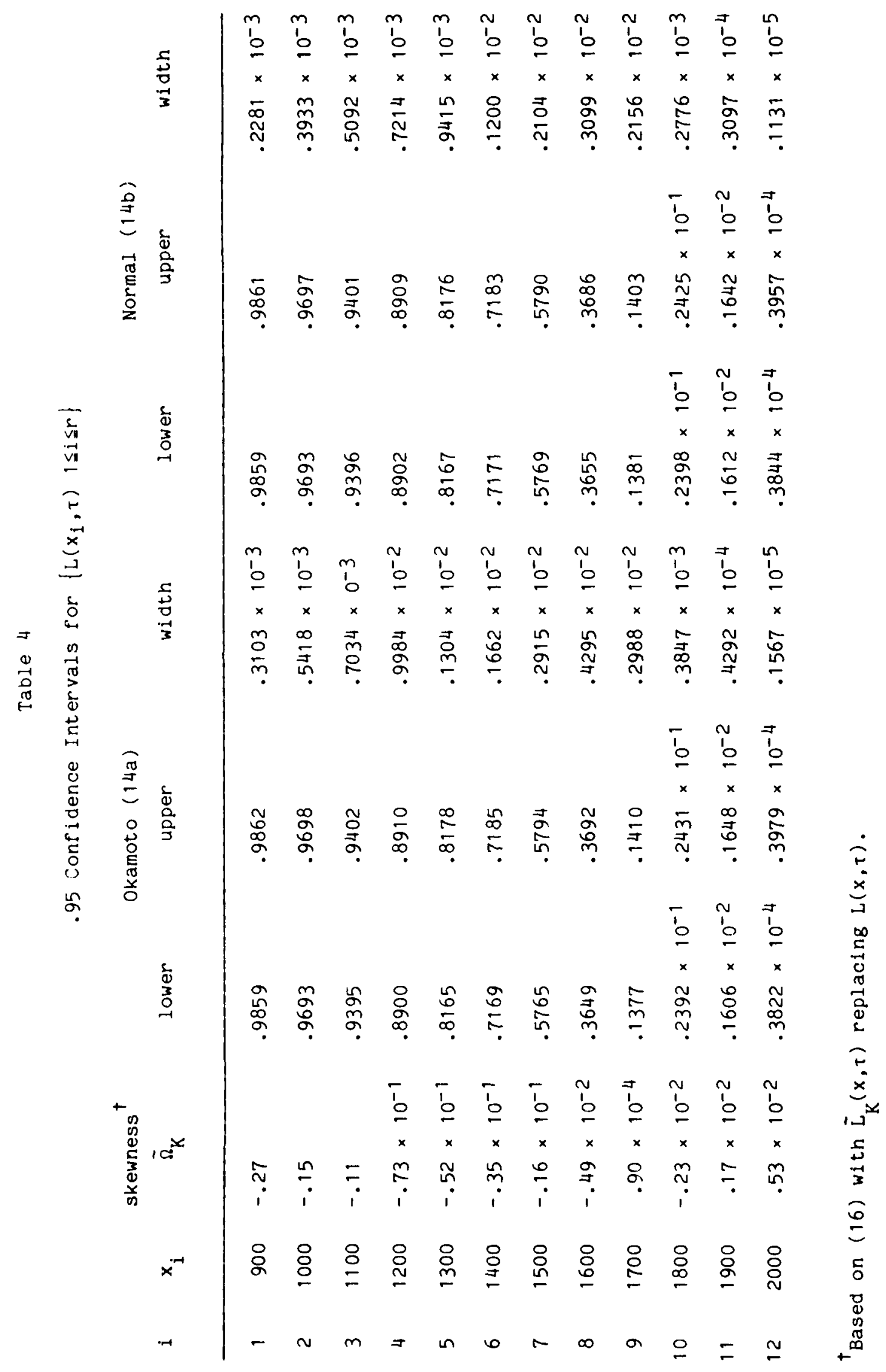




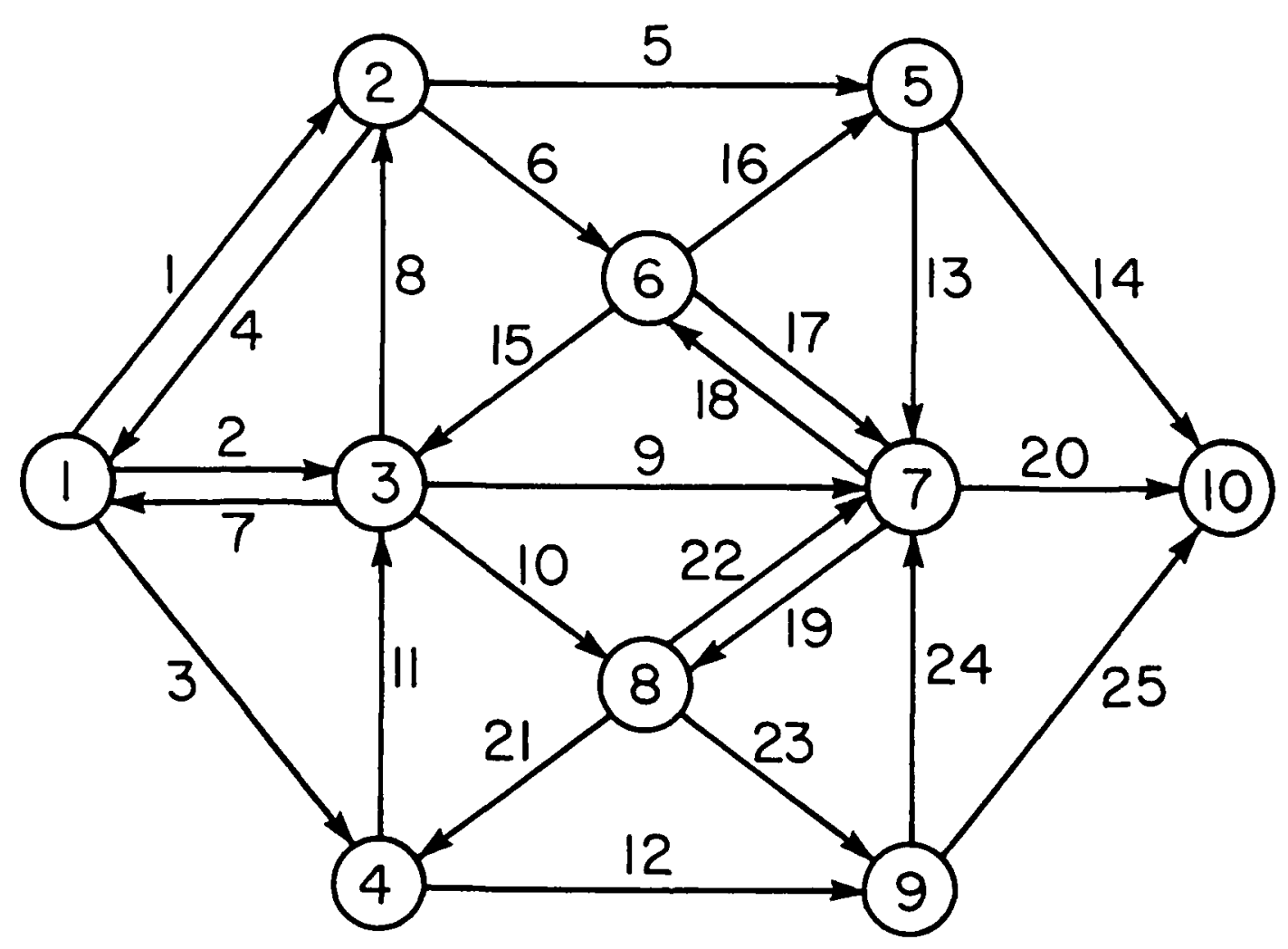

Fig. I: Network 


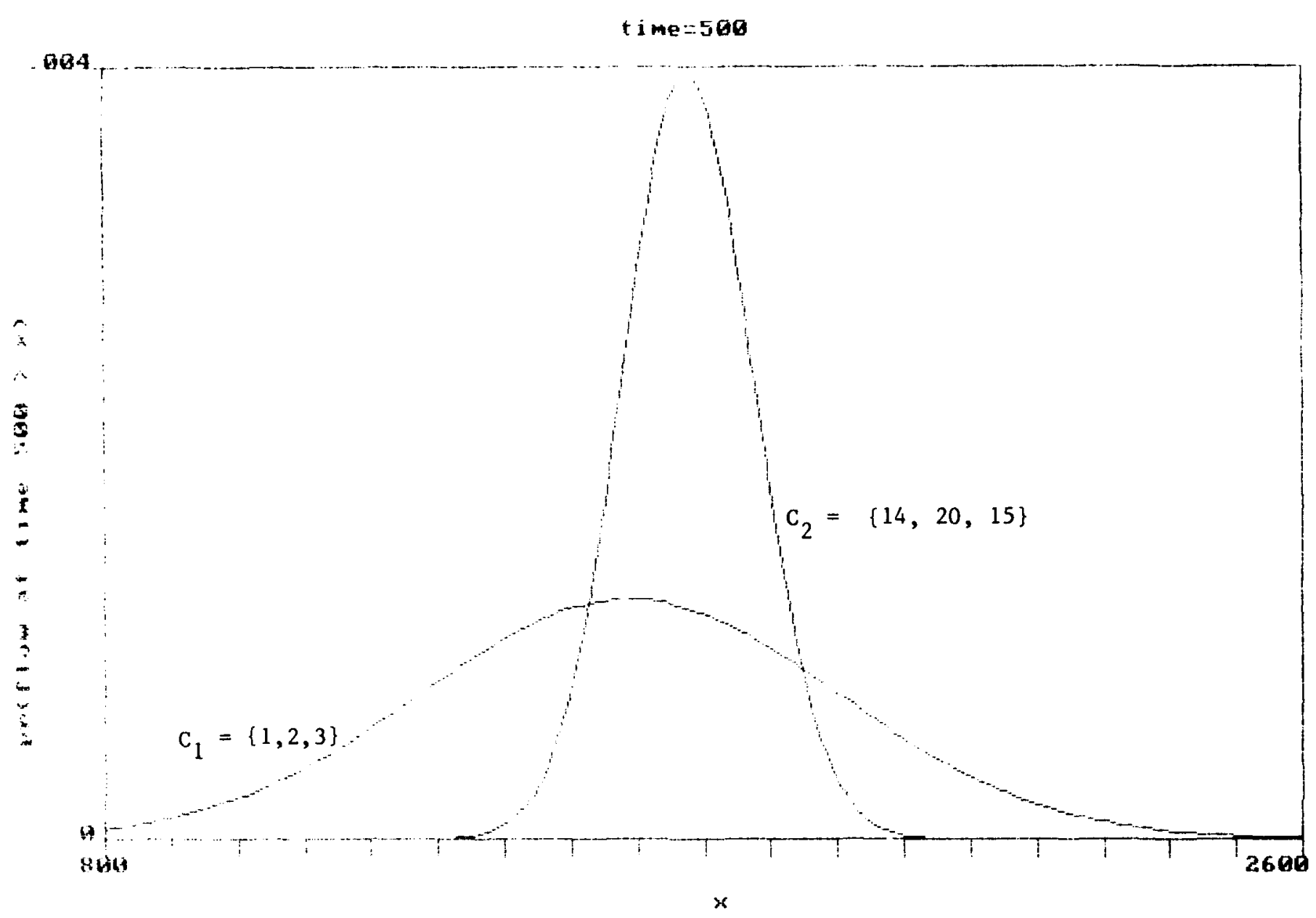

Fig. 2 Cutset Capacity Distributions 
Unclassifled

SECURiTTY CLASSIFICATION OF THIS PAGE (Whan Dote Entorod)

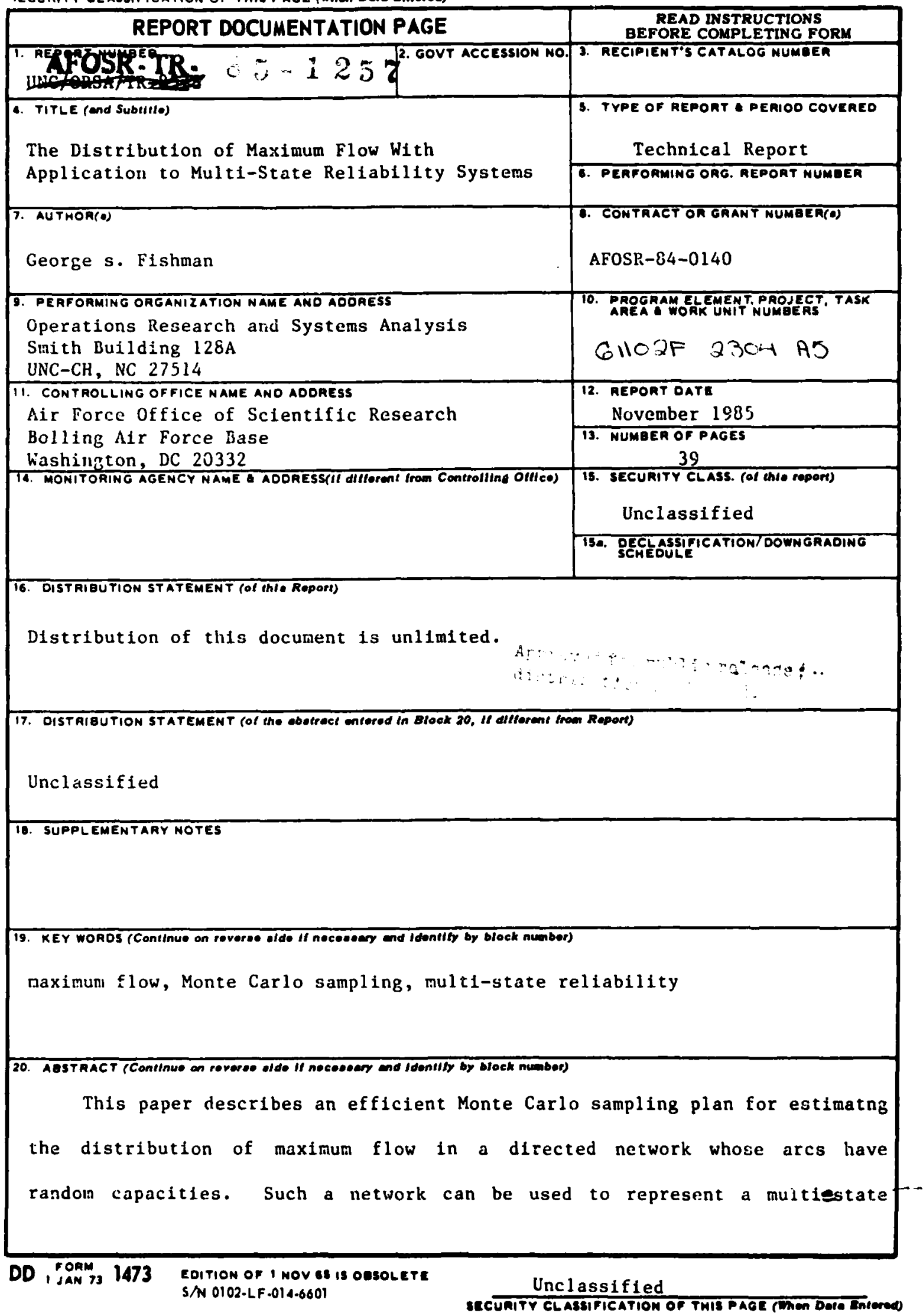


system whose multisstate components are subject to deterioration in capacity by random amounts at random points in time. The proposed sampling plan uses an easily computed a priori upper bound on the complementary distribution function to obtain an unbiased point estimator with smaller variance than crude Monte Carlo sampling allows. The paper also describes procedures for interval estimation and for assessing when the sampling experiment has achieved a specified accuracy. To facilitate sampling, the paper presents a characterization of deterioration based on cumulative processes, leading to the treatment of arc capacities as being multinormally distributed. A technique is described for checking the appropriateness of this model with regard to lower and upper bounds on capacity. A procedure is also described for deriving a confidence interval on the measure used to assess variance reduction. An example illustrates the sampling plan and a concise summary gives all steps needed to implement the plan. 
